Cahiers de philosophie de l'université de

\title{
Diderot, la perception des rapports : la musique prise entre réalisme et empirisme
}

Frédéric de Buzon

\section{(2) OpenEdition}

12 Journals

Édition électronique

URL : https://journals.openedition.org/cpuc/616

DOI : $10.4000 /$ cpuc.616

ISSN : 2677-6529

Éditeur

Presses universitaires de Caen

\section{Édition imprimée}

Date de publication : 31 décembre 2014

Pagination : 23-40

ISBN : 978-2-84133-512-1

ISSN : 1282-6545

\section{Référence électronique}

Frédéric de Buzon, «Diderot, la perception des rapports : la musique prise entre réalisme et empirisme », Cahiers de philosophie de l'université de Caen [En ligne], 51 | 2014, mis en ligne le 13 juin 2018, consulté le 04 février 2023. URL : http://journals.openedition.org/cpuc/616 ; DOI : https:// doi.org/10.4000/cpuc.616

\section{(c) (†) (8)}

Creative Commons - Attribution - Pas d'Utilisation Commerciale 4.0 International - CC BY-NC 4.0 https://creativecommons.org/licenses/by-nc/4.0/ 


\section{Diderot, la perception des rapports : la musique prise entre réalisme et empirisme}

$\mathrm{I}^{\mathrm{s}}$

L EST UNIVERSELLEMENT CONNU que Diderot a manifesté pendant toute sa carrière un intérêt soutenu pour les questions théoriques, pratiques et esthétiques ou critiques de la musique . "Questions théoriques", en ce qu'il s'intéresse à l'un des aspects fondamentaux de la pensée musicale, c'est-à-dire la théorie de l'harmonie, des consonances et des dissonances, et qu'il articule dans de nombreux textes cette question aux mathématiques, à la physique générale, et même à la physiologie de manière originale. "Questions pratiques», en ce qu'il rédige des Leçons de clavecin et principes d'harmonie, en reprenant la matière de l'enseignement du maître de musique d'Angélique Diderot, Anton Bemetzrieder, sans que l'on sache bien au demeurant la part précise que prend Diderot dans le livre achevé ${ }^{2}$, dont il indique n'être que le "traducteur " ${ }^{3}$; de même, on lui attribue (sans véritable preuve) une part dans la rédaction de la Démonstration du principe de l'harmonie de Rameau ${ }^{4}$. "Questions critiques", enfin, lorsque Diderot intervient dans la querelle des Bouffons, ou que nombre de textes «littéraires » sont imprégnés de remarques sur la musique et ses beautés.

Cette situation générale est comparable à celle d'autres figures majeures du milieu encyclopédique, comme Rousseau ou d'Alembert: l'un comme l'autre, à l'instar de Diderot, réfléchissent en philosophes et en savants sur la question des fondements de l'harmonie, prennent parti dans les

1. Voir l'anthologie présentée par Béatrice Durand-Sendrail: D. Diderot, Écrits sur la musique, B. Durand-Sendrail (éd.), Paris, J.-C. Lattès, 1987.

2. Voir sur ce point la grande étude de J. Gribenski, «À propos des Leçons de clavecin (1771): Diderot et Bemetzrieder ", Revue de musicologie, t. 66, $\mathrm{n}^{\circ}$ 2, 1980, p. 125-178, qui contient notamment une bibliographie très abondante des œuvres de Bemetzrieder.

3. Ibid., p. 135, selon une lettre à Sophie Volland de 1770.

4. C. Girdlestone, Jean-Philippe Rameau: sa vie, son œuvre [1957], Paris, Desclée de Brouwer, 1983, p. 491-492. 
querelles, discutent les positions théoriques et esthétiques de Jean-Philippe Rameau, et situent les explications qu'ils proposent dans l'horizon commun des travaux de physique et de mathématique comme ceux de Sauveur ou d'Euler; mais aussi dans l'horizon philosophique de Malebranche, de Leibniz, du père André, ou encore des Britanniques comme Hutcheson et Shaftesbury, et, plus lointainement sans doute, de l'Abrégé de musique de Descartes, dont la reprise par Rameau dans le Traité de l'harmonie signale l'importance. L'origine cartésienne - le Descartes du Compendium musicae tel qu'il est notamment connu par la traduction du père Poisson en 1668 - de certaines des thèses de Diderot est expressément mentionnée par Rousseau, notamment dans la réécriture de l'article "Consonance» qu'il insère dans le Dictionnaire de musique de 1768.

Rousseau écrit, pour caractériser la pensée musicale de Diderot:

Le philosophe qui nous a donné des principes d'Acoustique, laissant à part tous ces concours de vibrations et renouvelant sur ce point le système de Descartes, rend raison du plaisir que les Consonances font à l'oreille par la simplicité des rapports qui sont entre les sons qui les forment. Selon cet Auteur, et selon Descartes, le plaisir diminue à mesure que ces rapports deviennent plus composés et quand l'esprit ne les saisit plus, ce sont de véritables dissonances; ainsi c'est une opération de l'esprit qu'ils prennent pour le principe du sentiment de l'harmonies.

Comme on le sait, Rousseau préfère un autre système, celui d'Estève ${ }^{6}$. Mais c'est précisément le principe auquel Diderot donne la plus grande

5. J.-J. Rousseau, art. "Consonance», in Dictionnaire de musique [1768], in CEurres complètes, B. Gagnebin et M. Raymond (éd.), Paris, Gallimard (Bibliothèque de la Pléiade), t. V, 1995, p. 729. On notera que dans la version initiale de l'article, Rousseau évoquait de manière plus succincte la thèse de Diderot, sans nommer Descartes: «Un écrivain judicieux, qui nous a donné nouvellement des principes d'Acoustique, laissant à part tous ces concours de vibrations, a rendu raison du plaisir que les consonances font à l'oreille par la simplicité des rapports entre les sons qui les forment. Selon lui, le plaisir diminue à mesure que les rapports deviennent plus composés; et quand l'esprit ne les saisit plus, ce sont de véritables dissonances. Mais quoique cette doctrine s'accorde parfaitement avec le résultat des premières divisions harmoniques, quoiqu'elle soit très bien soutenue et qu'elle s'étende facilement à d'autres phénomènes qui se remarquent dans les beaux-arts, s'il se trouve qu'elle ne soit pas en tout d'accord avec l'expérience, s'il n'y a toujours une proportion exacte entre les rapports des sons et le degré de plaisir ou de peine dont ils nous affectent, je dis que cette hypothèse est fort vraisemblable, mais qu'il ne la faut pas regarder comme démontrée»; J.-J. Rousseau, art. "Consonance», in Encyclopédie ou Dictionnaire raisonné des sciences, des arts et des métiers, D. Diderot et J. Le Rond d'Alembert (éd.), Paris, Briasson, vol. IV, 1754, p. 41.

6. P. Estève, Nouvelle découverte du principe de l'harmonie avec un examen de ce que M. Rameau a publié sous le titre de "Démonstration de ce principe" [1752], A. Charrak (éd.), Fontenayaux-Roses, ENS Éditions (Theoria), 1997; et A. Charrak, Raison et Perception - Fonder l'harmonie au XVIII' siècle, Paris, J. Vrin, 2001. 
importance dans les textes théoriques d'esthétique, à savoir celui qui associe le plaisir esthétique, voire le beau lui-même, à la perception des rapports, et en situe le lieu dans la théorie de la musique, qui sera ici mis en question. Ce principe est formulé dans le premier des Mémoires sur différents sujets de mathématiques (1748), intitulé «Principes généraux d'acoustique ${ }^{7}$, et dans quelques textes proches, notamment la «Lettre à $\mathrm{M}^{\text {lle } * * *}{ }^{8}{ }^{8}$ et l'article «Beau» de l'Encyclopédie, pour mettre ce genre de réflexion en rapport avec les énoncés où Diderot, de manière paradoxale, semble donner à l'harmonie musicale une valeur paradigmatique dans la compréhension du plaisir et de la beauté. Il est clair que le «Mémoire» est le texte de référence des productions postérieures.

La structure du «Mémoire» de 1748 est complexe; il est délicat de déterminer la ou les questions qui le commandent, dans la relative aporie où demeure la question initiale, dont je vais aborder la formulation. En effet, même si le texte parait être un mémoire portant sur la physique du corps sonore et sa mathématisation (ce que confirme le titre de la table: «Principes généraux de la science du son avec une méthode singulière de fixer le son $[\ldots]$ », titre lui-même abrégé dans le corps de l'article en «Principes généraux d'acoustique» selon la terminologie nouvelle à l'époque) et si de nombreux développements ne concernent apparemment que la physique et les mathématiques (à ce point que des éditeurs modernes suppriment parfois, imprudemment, les passages jugés trop scientifiques), le point de départ est, à nos yeux du moins, esthétique et non scientifique: alors que Sauveur avait forgé le terme d'acoustique pour distinguer l'étude objective des phénomènes sonores indépendamment de leur valeur musicale 9 , Diderot semble ici confondre les deux domaines ou du moins les associer étroitement. Il n'y a en tout cas pas d'opposition entre un point de vue scientifique qui serait neutre esthétiquement et un point de vue proprement esthétique, mais leur juxtaposition et leur articulation est permanente.

7. D. Diderot, "Principes généraux d'acoustique», in Mémoires sur différents sujets de mathématiques, in Euvres complètes, H. Dieckmann et al. (éd.), Paris, Hermann, t. II, Philosophie et mathématique - Idées I, 1975, p. 235-281 (désormais "Mémoire»).

8. Cette lettre est une addition à la Lettre sur les sourds et muets (mai 1751), dont la destinataire est habituellement identifiée à $\mathrm{M}^{\text {lle }}$ de La Chaux. Voir la présentation de Laurent Versini: D. Diderot, Euvres, L. Versini (éd.), Paris, R. Laffont (Bouquins), t. IV, Esthétique-théâtre, 1996, p. 8 et p. 52.

9. Voir notamment l'Histoire de l'Académie royale des sciences, année 1700, Paris, G. Martin, J.-B. Coignard et H.-L. Guérin, 1761 [ $2^{\mathrm{e}}$ éd. ; $1^{\text {re }}$ éd. 1719], p. 134-135, et l'éloge de M. Sauveur, Histoire de l'Académie royale des sciences, année 1716, Paris, Imprimerie royale, 1718, p. 85; textes repris dans J. Sauveur, Collected Writings on Musical Acoustics, R. Rasch (éd.), Utrecht, The Diapason Press, 1984, p. 68-69 et p. 277. Le terme d'acoustique est conçu comme un analogon du terme "optique». 


\section{L'objet incertain du «Mémoire»}

Diderot entame le "Mémoire» par l'exposé de deux faits qui s'opposent. Avant d'en venir à la question technique qui sous-tend une partie de l'exposé, il faut décrire les présupposés initiaux.

D'une part, Diderot allègue l'expérience de la diversité des goûts, des cultures et des pratiques musicales qui font qu'un temps, qu'un peuple ou qu'un pays, voire un individu, jugent «admirables» des pièces que d'autres méprisent. L'argument relativiste, à lui seul, plaiderait pour l'idée, incarnée par la figure d'Aristoxène, qu'il n'y a pas de théorie de la musique au sens propre, mais seulement des convenances contingentes, ce que Diderot associe à un scepticisme; Diderot procède à l'instar de Mersenne, donnant un temps la parole à La Mothe Le Vayer pour mieux réfuter son scepticisme musical ${ }^{10}$. Dans cette perspective, les œuvres seraient le produit de l'arbitraire et du caprice, et dépendraient de coutumes non réglées rationnellement. Or, Diderot refuse absolument cette thèse sceptique, comme on le verra par la suite. Mais il lui faut aussi expliquer la diversité des goûts, ce qui constitue en soi un problème.

Le second fait initial est le suivant: Diderot allègue l'unanimité de la reconnaissance des intervalles consonants et associe cette unanimité à une certaine naturalité, suivant en cela la tendance, dominante à l'époque, de Rameau et la découverte des proportions dans le phénomène de la résonance ${ }^{11}$. Il dresse la liste «moderne» des consonances, celle de Zarlino, incluant les tierces et sixtes, et indique des dissonances (septièmes, secondes, tritons et autres), c'est-à-dire toutes les relations qui ne sont pas considérées comme consonantes, dans une filiation intellectuelle rapportée cette fois à Pythagore, même si ce dernier, comme l'indique Diderot, s'est trompé dans l'extension des consonances en les limitant à l'octave, à la quinte et à la quarte, et sur la manière d'apprécier les rapports. En ce qui concerne la liste des consonances, c'est naturellement, dans la théorie grecque, la méconnaissance de la valeur des tierces et des sixtes; en ce qui concerne la manière d'apprécier les rapports, l'erreur de Pythagore est sans doute la même que celle du père André que Diderot signale dans l'article «Beau » de l'Encyclopédie, à savoir la méconnaissance de l'origine empirique de ces notions. Or, c'est précisément sur ce point que Diderot rejette l'opinion du père André:

10. F. de La Mothe Le Vayer, Discours sceptique sur la musique, "Question 2 ", in M. Mersenne, Questions harmoniques, Paris, J. Villery, 1634; repris dans M. Mersenne, Questions inouïes, A. Pessel (éd.), Paris, Fayard, 1985, p. 141-165.

11. Voir sur ce point l'étude décisive d'A. Charrak, Raison et Perception..., citée plus haut. 
La seule chose qu'on pût désirer peut-être dans son ouvrage, c'était de développer l'origine des notions qui se trouvent en nous de rapport, d'ordre, de symétrie: car du ton sublime dont il parle de ces notions, on ne sait s'il les croit acquises $\&$ factices, ou s'il les croit innées: mais il faut ajouter en sa faveur que la manière de son ouvrage, plus oratoire encore que philosophique, l'éloignait de cette discussion, dans laquelle nous allons entrer ${ }^{12}$.

Toute la difficulté est d'associer la stabilité des rapports harmoniques et les variations empiriques, en répondant à la contrariété d'une double exigence.

Les deux faits initiaux indiquent une tendance à la variété sujette aux vicissitudes de l'histoire et de la culture et une tendance à l'uniformité des relations harmoniques admises comme plus agréables et dépendant de propriétés naturelles; autrement dit, Diderot paraît osciller entre rationalisme et empirisme, réalisme et scepticisme. Ramenée à des considérations plus contemporaines en esthétique, la question est alors de savoir si les propriétés esthétiques sont inhérentes aux objets mêmes ou au regard variable qui est porté sur eux, ou encore s'il faut une tierce solution.

On notera qu'une alternative semblable, résurgence du problème de l'Hippias majeur, détermine dès le début le problème de l'article «Beau»:

Tout le monde raisonne du beau: on l'admire dans les ouvrages de la nature; on l'exige dans les productions des arts: on accorde ou l'on refuse cette qualité à tout moment; cependant si l'on demande aux hommes du goût le plus sûr et le plus exquis quelle est sa nature, sa notion précise, sa véritable idée, son exacte définition; si c'est quelque chose d'absolu ou de relatif, s'il y a un beau essentiel, éternel, immuable, règle et modèle du beau subalterne ou s'il en est de la beauté comme des modes, on voit aussitôt les sentiments partagés et les uns avouent leur ignorance et les autres se jettent dans le scepticisme ${ }^{13}$.

Cette partie de l'article est certes de Diderot, mais comme les éditeurs l'ont signalé depuis longtemps, Diderot démarque et paraphrase strictement le début de l'Essai sur le beau du père André ${ }^{14}$, qui se situe comme on sait dans le strict prolongement de la pensée de Malebranche. Précisément, en contrepoint de la thèse musicale sceptique, le père André réfute lui aussi, après Mersenne, les arguments tirés de la relativité des goûts que l'on oppose à l'idée d'une théorie musicale scientifique munie de principes

12. D. Diderot, Recherches philosophiques sur l'origine et la nature du beau, in Euvres, t. IV, p. 97.

13. Ibid., p. 81 .

14. Y.-M. André, Essai sur le beau, Paris, H.-L. Guérin et J. Guérin, 1741. 
certains $^{15}$, au profit de l'affirmation d'un beau musical indépendant, et décliné selon les trois catégories sous lesquelles ce concept est pensé: beau musical essentiel (indépendant de la création - qui s'impose à Dieu comme créateur), beau musical naturel (il s'agit du monde créé) et beau musical artificiel (beau produit de l'activité humaine qui dépend toujours de l'essentiel et éternel). Il est clair, comme on le verra après, que ce beau musical est conçu par le père André comme a priori (même s'il n'est pas strictement inné, il dépend d'une autre source que la perception sensible) et déterminant l'expérience. Cette conception du beau et de ses niveaux ou espèces provient elle-même en droite ligne de Malebranche, qui écrit par exemple:

[I]l n'y a rien de beau ni rien d'aimable, que par rapport à la beauté essentielle, nécessaire et indépendante. Or cette beauté intelligible s'étant rendue sensible, devient encore en cet état la règle de la beauté et de la perfection ${ }^{16}$.

Mais, en même temps, apparaissent quelques difficultés: si Diderot se rattache, comme la plupart des théoriciens, à la conception de la musique attribuée classiquement à Ptolémée (qui marie une causalité formelle numérique à une causalité matérielle empirique, en conciliant Pythagore et Aristoxène), il y a une difficulté majeure quant à l'origine des idées de ce beau indépendant et de ces rapports, qui tient à la position radicalement empiriste de Diderot: il est amené à être en quelque sorte un pythagoricien empirique. Ce que je vais essayer de montrer.

Diderot admet les conséquences de la doctrine du père André dans l'appréciation esthétique, sans ses prémisses - à savoir la transposition de la conception malebranchienne de la raison éternelle et incréée aux motifs des jugements de goût. On peut rappeler la manière dont le père André traitait de ce beau essentiel:

15. «Il y a dans le monde une espèce de philosophes qui n'ont pas de la musique une idée si avantageuse, ou plutôt qui en ont une toute contraire. Ils prétendent que le sentiment est le seul juge de l'harmonie, que le plaisir de l'oreille est le seul Beau qu'on y doive chercher, que ce plaisir même dépend trop de l'opinion, du préjugé, des coutumes reçues, des habitudes acquises, pour pouvoir être assujetti à des règles certaines»; Y.-M. André, Essai sur le beau, p. 241.

16. N. Malebranche, Traité de la nature et de la grâce, I, XXIX, in EEuvres complètes, A. Robinet (éd.), Paris, J. Vrin, vol. V, G. Dreyfus (éd.), 1976, p. 41. Voir aussi, entre autres, les Méditations chrétiennes et métaphysiques: «Je conçois bien que la beauté de l'ordre est plus aimable que toutes les beautés sensibles. Oui je le conçois: mais en quoi consiste cette beauté, c'est ce que je ne puis découvrir. Plus je pense à elle, plus elle s'éloigne de moi, et lorsque je fais quelque effort pour la retenir, elle m'échappe et s'évanouit comme un fantôme qui disparaît à la lumière»; "IV $\mathrm{V}^{\mathrm{e}}$ Méditation», in Euvres complètes, vol. X, H. Gouhier et A. Robinet (éd.), 1986, p. 36. 
[P] endant que tous vos Concertants lisaient sur le papier leur tablature, vous lisiez aussi la vôtre écrite en notes éternelles et ineffaçables dans le grand livre de la raison, qui est ouvert à tous les esprits attentifs ${ }^{17}$.

Or, pour Diderot, il n'y a qu'une voie par laquelle les objets idéaux des mathématiques rentrent dans l'esprit, à savoir les sens, ou plutôt chaque sens pris isolément, sans l'hypothèse d'un sens commun - bien qu'il y ait une sorte de rationalité commune à ces sens: on peut concevoir une supposition où "chaque sens devînt géomètre ${ }^{18}$ et dans lequel se produirait en quelque sorte une intersubjectivité des sciences à l'intérieur même de la pensée d'un individu unique, dans la mesure où, dans cette «société» des cinq sens, «on parlerait de tout, [...] et l'on ne s'entendrait qu'en géométrie ${ }^{19}$. L'appel à une raison à la fois totalement extérieure à l'esprit et totalement intérieure, parce que parlant dans le silence des sens, Maître intérieur augustinien et malebranchien qui constitue l'argument majeur du père André, est évidemment une difficulté chez Diderot. Mais demeure, dans la structure même du «Mémoire», une trace nette de la conception du père André, antérieurement à la reprise explicite de cet auteur dans l'article «Beau».

En effet, c'est la division, propre au père André, des trois niveaux de beau qui organise l'articulation du problème chez Diderot, si l'on considère (1) que ce sont les rapports exprimés par des nombres qui définissent chez lui le beau essentiel et incréé; (2) que le beau naturel et créé, mais indépendant des esprits, est, comme chez le père André, la structure même des corps sonores et de la résonance, ce qui constitue l'objet de la plus grande partie du traité de Diderot; (3) qu'enfin le beau artificiel des pièces de musique dépend des deux précédents, mais laisse une place à l'arbitraire. Or, c'est exactement ce dont traite Diderot, sous la forme d'un principe de perception des rapports et d'une analyse du corps sonore; et, par ailleurs, il tend même à réduire la part d'arbitraire que le père André indique. En effet, de la double constatation initiale, variété et consensus (la seconde n'est pas réellement argumentée, alors que la première est illustrée de quelques exemples sommaires), Diderot tire une question, qui paraît quelque peu biaisée par rapport à ce qui précède, mais qui a la particularité de centrer le problème général sur la perception propre de la musique:

17. Y.-M. André, Essai sur le beau, p. 249-250.

18. D. Diderot, «Lettre à $\mathrm{M}^{\text {lle } * * *}$ ", in Euvres, t. IV, p. 52.

19. Ibid. 
Si ce consentement unanime avait un fondement réel dans la nature; si en effet tous les sons n'étaient pas également propres à former des consonances agréables, pourrait-on regarder la succession des sons et des consonances comme arbitraire? Quoi, les sons plairaient à l'oreille en se succédant indistinctement, tandis qu'il y aurait un choix délicat à faire pour arriver au même but, en les unissant? Cela n'est pas vraisemblable ${ }^{20}$.

L'interprétation de ces phrases n'est pas absolument évidente, ni même leur rapport strict et complet avec les deux faits initiaux. Diderot suggère manifestement que l'existence d'un fondement naturel du consensus portant sur les relations harmoniques (ce qui dispenserait d'autres preuves), revenant, par une sorte de conversion, à ce que «tous les sons [ne soient] pas également propres à former des consonances agréables» (i. e. des consonances tout court), interdit de penser que la succession «des sons et des consonances » soit arbitraire. Si le plaisir est le but poursuivi, tant dans les relations verticales du son que dans les relations horizontales, pourquoi penser qu'il y a de l'arbitraire possible dans la succession et un "choix délicat» dans l'union des sons? Cela revient ainsi, rapporté aux faits initiaux, à penser que la diversité historique et géographique de la musique provient des relations de succession (en bref, de ce qui relève de la mélodie), alors que subsistent des relations naturelles, celles qui constituent l'harmonie: de fait, se dessine une sorte de position moyenne entre la tendance italienne (privilège de la mélodie libre et expressive sur l'harmonie) et la tendance ramiste, qui fait de l'harmonie le principal moyen expressif de la musique. Et la question devient alors d'unifier les conditions du plaisir de l'harmonie et celles de la mélodie. On peut illustrer la question ainsi: si un intervalle de seconde (do-ré) n'est pas, dans l'hypothèse, jugé déplaisant à l'oreille lorsque les sons se succèdent, il constitue une dissonance lorsque les sons sont appréciés simultanément par l'oreille.

Ce point avait été traité dans la théorie musicale antérieurement à Diderot, par exemple lorsque Descartes jugeait que les degrés de la gamme - soit harmoniquement les secondes - sont utilisés comme un moyen permettant de modérer l'inégalité des consonances ${ }^{21}$, et constituent alors des transitions entre les consonances. En d'autres termes, dans une «musicologie» cartésienne, le degré de la gamme est un moyen de passer d'une consonance à l'autre aisément, et n'a pas de valeur esthétique par lui-même:

20. D. Diderot, «Mémoire», p. 236.

21. R. Descartes, Abrégé de musique. “Compendium musicae”, F. de Buzon (éd. et trad. fr.), Paris, PUF, 1987, p. 96-97. 
si l'on joue do-ré, on doit ajouter quelque chose de consonant avec le do initial pour terminer une phrase. On peut aussi, pour mieux apprécier la portée de cet argument, se placer à l'autre extrémité de l'histoire de la tonalité, en se rapportant à certaines prémisses théoriques exprimées à propos de l'atonalisme de Schoenberg et de la doctrine de l'émancipation de la dissonance. Le Schoenberg de Charles Rosen, dans lequel le musicologue rappelle l'évidence du caractère fondamental de la dissonance dans la composition classique comme moyen d'expression, signale que l'un des préjugés tenaces (dont, en un sens, Diderot se fait l'écho, mais pour le réfuter à sa manière) est qu'une condition pour qu'il y ait dissonance serait que deux notes doivent être jouées simultanément et non successivement ${ }^{22}$. Bien au contraire, ce qui fait la différence entre la consonance et la dissonance est que la consonance peut terminer une phrase (c'est ce que l'on nomme «cadence»), alors que la dissonance ne le peut faire sans laisser une impression d'inachèvement (ce que montre, par exemple, le motif des secondes mineures dans «Des pas sur la neige» de Debussy ${ }^{23}$ ). De fait, la relation verticale et la relation horizontale fonctionnent en réalité selon le même principe, que Diderot connaît fort bien, à savoir que la dissonance doit être "préparée et sauvée ${ }^{24}$. En ce sens, la question technique que pose Diderot désigne une difficulté interne à la théorie musicale elle-même et pour laquelle il trouve une réponse.

Mais ce n'est pas le seul cap que prend précisément le «Mémoire» dans l'ensemble de son parcours. En effet, s'il affirme, contre le scepticisme, la réalité de principes invariables et d'une théorie, le «Mémoire» traite moins de la théorie de la musique proprement dite que de ses fondements, dans la physique de la vibration et dans sa mathématisation. La question liminaire, celle de la disparité entre les relations verticales et horizontales, n'est finalement traitée que comme exemple du principe de perception des rapports. Il faut alors revenir sur ce principe lui-même, en considérant qu'il joue le rôle d'absolu qu'il avait dans l'esthétique du père André, mais dénué de toute transcendance ou indépendance des esprits singuliers. Ainsi, l'examen de ce principe pourra montrer à quel point l'esthétique de Diderot est tendue entre un pôle empiriste et un pôle réaliste, même si elle suggère une solution au problème de la relativité culturelle des goûts.

22. C. Rosen, Schoenberg, P.-E. Will (trad. fr.), Paris, Minuit, 1980, chap. II, p. 31.

23. C. Debussy, Préludes, Livre I, $\mathrm{n}^{\circ} 6$.

24. D. Diderot, «Mémoire», p. 257 ; J.-P. Rameau, Traité de l'harmonie réduite à ses principes naturels, Livre IV, chap. XV, Paris, J.-B.-C. Ballard, 1722, p. 411-416; J. Le Rond d'Alembert, Éléments de musique théorique et pratique suivant les principes de M. Rameau, Livre I, chap. XVI, Lyon, J.-M. Bruyset, 1762, p. 78 ; J.-J. Rousseau, art. «Sauver», in Dictionnaire de musique, p. 1032. 


\section{D'André à Diderot, la constitution d'un malebranchisme empiriste: les rapports et leur perception}

Si le principe de perception des rapports est généralisé à tous les sens et tous les sensibles, il est d'abord explicité dans la théorie de la musique. Rappelons brièvement qu'antérieurement, il y avait deux types d'approche: les consonances étaient expliquées soit par les perfections des nombres, de Pythagore à Zarlino (mais on ne sait pas au juste comment elles sont comprises par le sens), soit par la coïncidence des chocs ou le concours des vibrations, comme chez Beeckman, Galilée et Mersenne: la perfection est plus grande à mesure que l'on se rapproche de l'unisson. Mais outre que ce principe est démenti par l'expérience du tempérament ${ }^{25}$, pourquoi en est-il ainsi ?

Le principe de Diderot apparaît alors comme une alternative à la solution par le concours des vibrations, qui s'était imposée sur les ruines de la solution purement mathématique valable jusqu'à la Renaissance, et qui est un exemple de plus du passage de la théorie de la perception comme réception d'images à une théorie causale. Il est annoncé dans le paragraphe II, comme devant être démontré par la suite; puis il est discuté de près dans le paragraphe VI, à propos de l'uniformité des sons. À vrai dire, il ne paraît explicitement démontré que par la négative. La preuve, tout indirecte, est tirée du déplaisir de la dissonance. Mais peut-on croire qu'elle est totalement convaincante?

Le chagrin de l'organe naît de ce que le défaut d'isochronisme dans les vibrations, rendant le rapport d'un son variable, il ne sait en quelle raison ce son qui le frappe est à celui qui le précède, l'accompagne ou le suit. Ce qui démontre que le plaisir musical consiste dans la perception des rapports de son ${ }^{26}$.

Le paragraphe II propose une définition:

Un objet est plus ou moins compliqué selon qu'il offre à l'esprit plus ou moins de rapports à saisir et à combiner en même temps et selon que ces rapports sont plus ou moins éloignés ${ }^{27}$.

25. Le tempérament désigne l'ajustement des intervalles dans les divers systèmes d'accord des instruments à clavier, venant de l'impossibilité de faire correspondre exactement la série des quintes justes avec celle des octaves. On distingue ainsi le tempérament mésotonique ou irrégulier du tempérament égal, ce dernier facilitant les transpositions - Le Clavecin bien tempéré de Bach en étant le paradigme. Le mouvement dit «baroqueux» a travaillé à montrer l'importance du tempérament pour rendre la coloration particulière de la musique antérieure au XIX ${ }^{\mathrm{e}}$ siècle. Si l'hypothèse du concours des vibrations est juste, alors il est difficile d'expliquer qu'un intervalle soit dissonant dans un tempérament, et consonant dans un autre. A. Baynes, art. "Tempérament", in Dictionnaire encyclopédique de la musique [1983], D. Arnold (dir.), Paris, R. Laffont (Bouquins), 1988.

26. D. Diderot, «Mémoire», p. 255-256.

27. Ibid., p. 236 . 
D’une manière générale, l'esprit (ou l'oreille) estime, combine, compare, distingue ces rapports; c'est dans la capacité à réaliser ces différentes opérations que Diderot compare les esprits entre eux et répond définitivement à l'objection relativiste contre la théorie musicale. Par exemple, le même paysan qui avait eu du plaisir à entendre deux parties séparées de flûte les juge insupportables lorsque celles-ci sont jouées ensemble, parce qu'il y a davantage de rapports à combiner, et ainsi se comprend le paradoxe initial.

Du point de vue de l'histoire de la théorie musicale, on a raison de voir dans ce passage, comme l'a suggéré Rousseau, un rappel de certaines thèses cartésiennes. Dans le Dictionnaire de musique, comme on l'a vu, Rousseau rapproche la thèse de Diderot de celle de Descartes. Quel Descartes? Sans reprendre tout dans le détail, on peut confirmer l'exactitude du propos, qui témoigne de la permanence de la réflexion cartésienne sur la musique dans la théorie du XVIII siècle. La définition de la complexité de l'objet musical est établie dans les «Praenotanda» du Compendium musicae:

$4^{\circ}$ L'objet est plus facilement perçu par le sens quand la différence de ses parties est moindre.

$5^{\circ}$ Nous disons que les parties de l'objet entier sont moins différentes entre elles quand elles entretiennent une plus grande proportion ${ }^{28}$.

L'application de ces remarques aux objets musicaux (mesure et hauteur) se fait en utilisant des proportions arithmétiques, et en limitant les nombres sonores, c'est-à-dire, dans la conception propre à Descartes, les facteurs premiers des grandeurs mises en rapport, à 2,3 et 5 . On peut remarquer que le concept de nombre sonore prend chez Descartes un sens nouveau ${ }^{29}$, qui devient déterminant. On comprend ainsi la portée de l'exemple numérique pris par Diderot dans le paragraphe II:

Quand on saura comment l'oreille estime les intervalles des sons, on ne balancera point à prononcer qu'elle apercevra plus facilement le rapport de deux sons qui sont l'un à l'autre comme 1 à 2 que s'ils étaient entre eux comme 18 à $19^{30}$.

28. R. Descartes, Abrégé de musique..., p. 56.

29. "Cette notion [de nombre sonore] recouvre depuis les Platoniciens jusqu'à Zarlino une réalité musicale bien précise, étayée par une vision globale de l'univers. Le nombre sonore était la matérialisation, dans la musique, des proportions générales de l'univers. Descartes, dans son souci de séparer le discours musical de toute spéculation mystico-métaphysique, vide ce terme de sa portée cosmologique pour en faire un simple synonyme de consonance, sans plus aucune référence à la chaîne des savoirs telle qu'elle était en vigueur chez les Pythagoriciens»; B. Van Wymeersch, Descartes et l'évolution de l'esthétique musicale, Sprimont, P. Mardaga, 1999, p. 112.

30. D. Diderot, «Mémoire», p. 237. 
Descartes, certes, opposait dans le «Praenotandum 6 » la saisie d'une proportion arithmétique simple (par exemple 2, 3, 4) à la saisie d'une proportion géométrique entre les deux mêmes extrêmes, qui serait 2, 8 , 4 et dont les termes sont incommensurables. Mais, même exprimée en nombres entiers, la proportion doit être très simple. L'argument porte, comme chez Descartes, non sur une beauté intrinsèque des nombres, mais sur une plus ou moins grande facilité à percevoir les rapports entre des quantités mesurées par ces nombres. Mais, à cette première détermination cartésienne complexe en elle-même, il faut ajouter deux autres éléments.

(1) Dans le droit fil de la pensée malebranchienne, le père André, qui cite à l'occasion le "Grand Descartes", situe le beau essentiel en musique dans les nombres sonores:

C'est-à-dire, Monsieur, que pendant que tant de voix \& d'instruments sonores vous frappaient l'oreille par des accords agréables, vous sentiez au dedans de vous-même un Maître de Musique intérieur qui battait la mesure, si j'ose ainsi parler, pour vous en marquer la justesse; qui vous en découvrait le principe dans une lumière supérieure aux sens; dans l'idée de l'ordre, la beauté de l'ordonnance du dessein de la pièce; dans l'idée des nombres sonores, la règle des proportions \& des progressions harmoniques, dont ils sont des images essentielles [... $]^{31}$.

La conception du nombre chez le jeune Descartes est certes à peu près inconnue - sinon qu'il maintient la distinction classique des deux genres de quantité - mais en tout cas le nombre désigne dans le Compendium musicae les manières par lesquelles on peut décomposer tout objet musical formé de parties. De ce point de vue, on peut identifier les nombres d'André aux proportions de Diderot. C'est bien, comme le montre en particulier le paragraphe $\mathrm{V}$, ce qui permet de comprendre la différence de hauteur des sons par le rapport du nombre de vibrations dans un temps donné. C'est précisément l'aperception de ce rapport qui a charge de produire le plaisir musical. Par rapport au père André, il y a un décalage de vocabulaire: on passe bien du beau au plaisant. Mais le plus important est que c'est la prise en considération du nombre de vibrations par l'esprit qui détermine le sentiment, et non le nombre tout seul ou le seul effet mécanique de coïncidence des chocs seuls. L'histoire de cette question est encore à écrire, l'argument bien connu des lettres de Leibniz à Goldbach étant précédé de considérations analogues chez Mersenne ${ }^{32}$.

31. Y.-M. André, Essai sur le beau, p. 248-249. Voir aussi p. 219.

32. Voir notamment la «Lettre de Leibniz à Christian Goldbach du 17 avril 1712 », traduite et présentée par F. de Buzon, Philosophie, ${ }^{\circ}$ 59, 1998, p. 11. Le précédent chez Mersenne de la thèse du calcul occulte a été identifié par R. Damann puis par U. Leisinger, qui renvoient à un passage de l'Harmonie universelle. 
(2) Se pose alors un problème qui est relativement classique, lié aux difficultés entraînées par la notion de perception. Diderot l'introduit dans le paragraphe VI, après le passage précédemment cité concernant la démonstration du principe, dans l' « Objection » faisant suite à la «Remarque»; la «Remarque» généralise le principe (vrai dans l'architecture, la morale, la poésie, dans tous les arts et toutes les sciences), ce qui signifie, comme chez Malebranche, que toute vérité est la perception d'un rapport, selon les différents domaines, rapport de grandeur ou rapport de perfection. Mais alors, demande l'" Objection", si le "plaisir des accords consiste dans la perception des rapports", faut-il connaitre ces rapports comme un mathématicien pour éprouver du plaisir? Un contre-exemple immédiat peut être produit, puisque l'on peut jouir du plaisir de la quinte sans savoir que les vibrations sont comme 2 à 3 par rapport au son fondamental, ni même sans percevoir distinctement ce rapport.

Ce qui revient à articuler, voire à opposer - comme Malebranche puis André -, une connaissance claire à un sentiment confus. Diderot demande:

Mais comment se peut-il faire, dira-t-on, que le plaisir des accords consiste dans la perception des rapports des sons? La connaissance de ces rapports accompagne-t-elle toujours la sensation? C'est ce qu'il paraît difficile d'admettre; car combien de gens, dont l'oreille est très délicate, ignorent quel est le rapport des vibrations qui forment la quinte ou l'octave, à celles qui donnent le son fondamental. L'âme a-t-elle ces connaissances sans s'en apercevoir, à peu près comme elle estime la grandeur et la distance des objets sans la moindre notion de géométrie, quoiqu'une espèce de Trigonométrie naturelle et secrète paraisse entrer pour beaucoup dans le jugement qu'elle en porte?

Nous ne déciderons rien là-dessus; nous nous contenterons d'observer qu'il est d'expérience que les accords les plus parfaits sont formés par les sons qui ont entre eux les rapports les plus simples; que ces rapports peuvent affecter notre âme de deux manières, par sentiment ou par perception, et qu'ils n'affectent peut-être la plupart des hommes que de la première manière ${ }^{33}$.

Les éléments de la «Réponse» suggèrent bien les deux voies décrites par Malebranche si l'on donne à la perception le rôle de l'idée claire. Ce point est renforcé par l'article «Beau » de l'Encyclopédie dans lequel la fonction du sentiment est d'éveiller l'idée de rapport, mais sans que cet éveil soit l'occasion d'une relation à un espace intelligible transcendant. Le rapport est défini généralement comme une "opération de l'entendement qui

33. D. Diderot, «Mémoire», p. 257-258. 
considère soit un être, soit une qualité, en tant que cet être ou cette qualité suppose l'existence d'un autre être ou d'une autre qualité » ${ }^{34}$. Définition « ontologique» du rapport, que Diderot répartit en rapports réels, aperçus et intellectuels ou fictifs. La préférence qu'il donne aux rapports réels sur les rapports aperçus et fictifs confirme chez lui le souci de la recherche d'une objectivité du beau, ou plus exactement, une objectivité et une réalité des traits qui, dans la sensation, provoquent l'idée de beauté: de ce point de vue, le beau est certes dans le jugement, mais en tant qu'il est provoqué par des causes qui doivent posséder en elles-mêmes les rapports beaux et partant plaisants ${ }^{35}$.

Du côté du sujet percevant, Diderot retrouve alors des arguments bien connus de Descartes et de Malebranche, mais il modifie en grande partie l'héritage malebranchiste. Comme on le sait, Descartes décrit dans La Dioptrique la saisie des distances et grandeurs par une sorte de géométrie naturelle ${ }^{36}$; Malebranche indique que cette géométrie, nécessaire à la saisie des propriétés visibles, n'est pas réalisable par l'esprit fini seul et qu'elle se fait dans l'esprit, en lui, sans lui et malgré lui ${ }^{37}$; l'objection précédente donne comme solution possible au problème un analogon acoustique à cette trigonométrie naturelle et secrète de l'âme, avant même que d'être généralisée aux autres sens.

Mais la thèse de Malebranche, ainsi que sa reprise par le père André, supposent deux éléments: d'une part, un réalisme des notions, et en particulier celui des nombres, et d'autre part, l'action directe d'une puissance supérieure à l'esprit, indépendante de la connaissance rationnelle, susceptible de compenser le défaut d'attention, et plus généralement, la finitude de l'esprit. Diderot ne peut partager aucune de ces deux présuppositions.

Il affirme en effet une conception empiriste du nombre. Ainsi, dans sa


distribué en autant de parties pensantes que nous avons de sens ${ }^{38}$ (c'està-dire la supposition voisine de celle de Condillac, et que paraît refuser la

34. D. Diderot, art. «Beau (Métaphysique)», in Encyclopédie ou Dictionnaire raisonné des sciences, des arts et des métiers, D. Diderot et J. Le Rond d'Alembert (éd.); édition en ligne: R. Morrissey (éd.), University of Chicago, ARTFL Encyclopédie Project, 2013, disponible à cette adresse: http://encyclopedie.uchicago.edu/.

35. Voir dans ce recueil le texte de Carole Talon-Hugon.

36. R. Descartes, La Dioptrique, "Discours VI», in Euvres, C. Adam et P. Tannery (éd.), Paris, J. Vrin, t. VI, 1965, p. 137 (cette édition sera désormais désignée par la mention AT suivie de la tomaison); Traité de l'homme, AT XI, 1967, p. 160.

37. «C'est pour cela que j'ai appelé naturels ces sortes de jugements, pour marquer qu'ils se font en nous, sans nous, et même malgré nous"; N. Malebranche, De la recherche de la vérité, IX, in Euvres complètes, vol. I, G. Rodis-Lewis (éd.), 1972, p. 121.

38. D. Diderot, «Lettre à $M^{\text {lle *** } », ~ p . ~} 52$. 
correspondante de Diderot) où, comme on l'a vu, chaque sens deviendrait géomètre, permet d'assigner une origine empirique aux notions abstraites des nombres, telles les «mathématiques pures» qui entrent «dans notre âme par tous les sens» et constituent une sorte de société des sens par les seules abstractions mathématiques. Le propos, mi-sérieux mi-plaisant, a pour but manifeste de faire entrer des notions liées au goût, à l'odorat et au toucher, dans l'ensemble de ce qui est dit de la vue - où la mathématique paraît plus évidente. En tout cas, c'est sur le fond de cette théorie de la perception et du nombre associé à chaque perception que peut se déployer l'ensemble de cette esthétique: lorsque nous percevons une, deux, trois choses par n'importe quel sens, nous avons en même temps la notion de un, deux, trois ${ }^{39}$, et, à partir de là, une communauté de nombres et donc de proportions est possible. Mais un, deux et trois proviennent de l'acte même de dénombrer. Le nombre, l'arithmétique qui se construit sur lui, et par extension l'algèbre tout entière, ont leur origine dans l'expérience du dénombrement:

Les expériences fréquentes qui nous constatent l'existence des êtres ou de leurs qualités sensibles, nous conduisent en même temps à la notion abstraite des nombres; et quand le toucher par exemple dira: «j'ai saisi deux globes, un cylindre", de deux choses l'une: ou il ne s'entendra pas; ou avec la notion de globe et de cylindre, il aura celle des nombres un et deux qu'il pourra séparer par abstraction des corps auxquels il les appliquait et se former un objet de méditation et de calculs; de calculs arithmétiques si les symboles de ses notions numériques ne désignent ensemble ou séparément qu'une collection d'unités déterminée; de calculs algébriques, si, plus généralement, ils s'étendent chacun indéterminément à toute collection d'unités ${ }^{40}$.

On voit alors parfaitement ce qui, en dépit de grandes similitudes, oppose Diderot à Malebranche dans la question de l'origine empirique des nombres. Pour Malebranche, les nombres sont logiquement antérieurs au dénombrement, puisqu'ils sont des éléments de la raison éternelle à laquelle participent les êtres raisonnables ${ }^{41}$; pour Diderot, au contraire,

\footnotetext{
39. Ibid., p. 53 .

40. Ibid.

41. Ce qui oppose Malebranche à Descartes, puisque, pour ce dernier, les nombres, comme les universaux, ne sont que des modi cogitandi, des manières de penser (cf. Principes de la philosophie, I, art. 58, AT VIII, 1964, p. 27 ; trad. fr. AT IX, 1964, p. 50). Thèse que l'on opposera, par exemple, à cette proposition de Malebranche: "Assurément, quand je pense à un cercle, à un nombre, à l'être ou à l'infini, à tel être fini, j'aperçois des réalités "; Entretiens sur la métaphysique et sur la religion, «Premier entretien", $\$ 4$, in Euvres complètes, vol. XII-XIII, A. Robinet (éd.), 1984, p. 35.
} 
ils en sont l'effet, ce que montre parfaitement le passage cité, et que l'on peut associer aux conceptions empiristes du nombre ${ }^{42}$.

Le second présupposé malebranchiste, repris par le père André, et que refuse Diderot, est la conséquence noétique du réalisme des nombres et des harmonies. La thèse de Malebranche et d'André fonctionne ainsi: la partition intelligible est certes perceptible à l'esprit attentif, comme on l'a vu plus haut, mais ce n'est pas cette intelligibilité qui plait à l'esprit grossier qui ne dispose que de ses oreilles pour saisir les beautés, coupé dans les faits de la raison universelle. Si cet esprit grossier prend du plaisir, c'est parce qu'il éprouve dans son propre corps l'effet de cette harmonie sous forme d'un sentiment confus. Il ne le conçoit pas, mais le subit. Cet argument est évidemment pour Malebranche une preuve de l'inefficacité des causes secondes et un argument en faveur de la croyance en l'effectivité d'un être pouvant donner à l'esprit fini les sentiments qu'il se donnerait s'il avait la possibilité d'accéder sans effort à la beauté intelligible. Mais Diderot ne peut en aucun cas admettre cet argument, dont l'engagement théologique est manifeste. D'où viennent alors le plaisir et la diversité des goûts? Leur évidence, aux yeux de Diderot, ne compromet en rien le principe de la perception des rapports, en tant que ces rapports doivent être dans la cause externe de la sensation, mais le confirme, car il faut aussi quelque chose qui soit présent dans le corps sentant, ce que Diderot nomme dans la fin de la «Lettre à $\mathrm{M}^{\mathrm{lle} * * *}$ », des corps harmoniques:

En musique, le plaisir de la sensation dépend d'une disposition particulière non seulement de l'oreille, mais aussi de tout le système des nerfs. S'il y a des têtes sonnantes, il y a aussi des corps que j'appellerais volontiers harmoniques, des hommes qui en toutes les fibres oscillent avec tant de promptitude et de vivacité que, sur l'expérience des mouvements violents que l'harmonie leur cause, ils sentent la possibilité de mouvements plus violents encore et atteignent à l'idée d'une musique qui les ferait mourir de plaisir ${ }^{43}$.

À ces variétés physiques correspondent les cinquièmes conjectures des Pensées sur l'interprétation de la nature ${ }^{44}(\$ 36)$, généralisant à tous

42. Sur la conception empiriste du nombre, voir l'exposé critique remarquable de Louis Couturat, dont la conclusion établit que cette théorie «se borne, en effet, à décrire l'acte psychologique du dénombrement, sans en rechercher le principe et la raison d'être; elle constate le fait matériel et positif, à savoir que l'on compte les objets en disant (mentalement): "Un, deux, trois, quatre..." mais elle ne peut l'expliquer, car elle en ignore la signification logique et la valeur rationnelle. Elle ne voit dans cette opération que le côté extérieur et superficiel et ne s'occupe pas des conditions idéales qui la rendent possible et intelligible [...] »; L. Couturat, De l'infini mathématique, Paris, F. Alcan, 1896, p. 331.

43. D. Diderot, «Lettre à $M^{\text {lle } * * *}$ », p. 60.

44. D. Diderot, Pensées sur l'interprétation de la nature, in CEuvres, t. I, Philosophie, 1994, p. 575. 
les corps le même paradigme physique, permettant de penser à la fois la réalité des rapports des nombres, leur rationalité et le plaisir des corps, augmenté par le plaisir intellectuel, sans autre hypothèse que ces corps mêmes.

Frédéric DE Buzon

Université de Strasbourg

GREAM 